\title{
Estrategia de restauración ambiental en la cuenca sur del río Manzanares de Madrid
}

\author{
Environmental restoration strategy in the \\ Manzanares' south watershed of Madrid
}

Resumen

Autores:

Miguel Ángel Ajuriaguerra Escudero* miguelangel.ajuriaguerra@urjc.es Alba Ramírez Saiz** albaramirezs@outlook.com

* Universidad Rey Juan Carlos
** Universidad Politécnica de Madrid

España

Recibido: 30/Sep/2020 Aceptado: 23/Mar/2021
$\mathrm{L}$ cuenca sur del río Manzanares en Madrid experimenta un proceso de restauración ambiental englobado dentro del proyecto Parque Lineal. estas mejoras se contraponen con la sensación de abandono institucional que experimentan los vecinos de los barrios interiores, viéndose alejados de las mejoras de regeneración ambiental del Manzanares. El objetivo de este artículo es identificar las problemáticas principales relacionadas con la accesibilidad, la usabilidad y la salubridad del Parque Lineal. Además, se exponen las acciones necesarias para alcanzar una propuesta estratégica integral que favorezca la consolidación del distrito mediante elementos de infraestructura verde que faciliten las relaciones de movilidad y usabilidad entre las áreas interiores y el perímetro de la restauración ambiental del Manzanares.

Palabras clave: planificación urbana; estrategia; recuperación ambiental; Villaverde-Madrid; tratamiento de aguas.

\section{Abstract:}

The south watershed of the Manzanares river in Madrid is experiencing an environmental restoration process linked to the Parque Lineal project. This Strategic Planning represents a substantial improvement in the quality of life of the Villaverde's citizens near the river park. However, these improvements are not fulfilling the neighbor's feeling of institutional abandonment, especially by residents of inner neighborhoods. This population is away from the improvements that are experienced as for the regeneration of the Manzanares river.

This paper identifies the main problems related to accessibility, usability, and health of the Linear Park. And the actions necessary to achieve a comprehensive strategic proposal that favors the consolidation of the district through backbone elements that facilitate the mobility and usability relations between the interior areas and the environmental restoration of the Manzanares river.

Keywords: urban planning; strategic planning; environmental restoration; Villaverde-Madrid; water recovery. 


\section{Introducción}

La denominación de la cuenca sur o cuenca baja del río Manzanares, ubicado en Madrid, como se muestra en la Figura 1, corresponde a los tramos que circulan por los distritos de Usera, Vallecas y Villaverde, así como por los municipios de la corona sur madrileña de Rivas y Getafe. Dichos distritos son tradicionalmente áreas residenciales de sesgo socioeconómico medio y bajo rodeados por el desmantelamiento industrial madrileño y por el asentamiento de nuevos polos industriales (Ajuriaguerra, Braojos Bueno y López Parejo, 2019). En concreto, los distritos de Usera y Villaverde se ubican en un entorno privilegiado al estar en contacto directo con el río Manzanares y sus procesos de renaturalización. Sin embargo, se encuentran enmarcados dentro de las dos diagonales de la pobreza y del río, como se ve en la Figura 2.

Con respecto al estado del río, en la actualidad existe un plan de reurbanización que busca conectar los espacios dotacionales de MadridRío y del Parque Lineal del Manzanares. En este artículo se analizan las estrategias seguidas y las problemáticas existentes para lograr su reurbanización y, específicamente, se centra en los dos primeros tramos que resultan clave en la reurbanización ambiental y social del conjunto de la cuenca. Sin embargo, es importante destacar las diferencias existentes entre MadridRío y el Parque Lineal. Mientras que el primero es una acción consolidada de éxito con respecto a la regeneración urbana y social de los distritos que atraviesa (Martín Robles y Pancorbo Crespo, 2014), el segundo carece de la cohesión social y ambiental, no solo con respecto a MadridRío, sino también con el resto de los barrios y municipios por los que transcurre (Martínez Escobar, 2019), a pesar de haber recibido numerosas propuestas de actuación y mejora.

A su vez, es importante considerar que MadridRío favoreció la recuperación del río por parte de los ecosistemas del margen fluvial tras el soterramiento de la M-30 (primera corona de vía rápida de circunvalación, anteriormente paralela al río). Su reurbanización abarcó un gran tramo continuo de la ribera (Orueta, 2015), generando una gran infraestructura verde que conectara todo el sur de la almendra central madrileña. No obstante, las actuaciones del Parque Lineal no representan una continuación de la ribera, a pesar de contar con una pasarela de conexión y dotaciones singulares a lo largo de los primeros tramos, tal y como se aprecia en la Figura 3. Por ello, es en esta desconexión entre el tramo de Madrid-Río y el tramo del Parque Lineal, donde se encuentra la oportunidad de generar una conexión real que permita tanto el tránsito como la movilidad, usabilidad, accesibilidad y renaturalización de un entorno de alto valor ecológico. Todo ello resulta fundamental para revitalizar los barrios más desfavorecidos de los distritos de Usera y Villaverde, proporcionando oportunidades empresariales reales que favorezcan la mejora socioeconómica de sus habitantes.

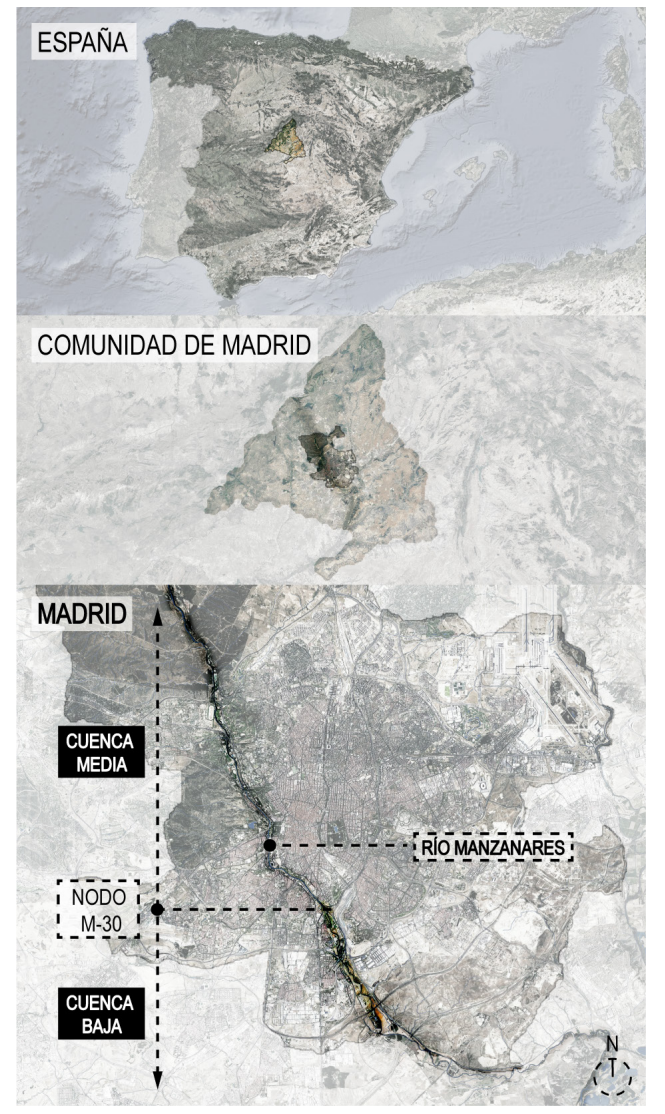

Figura 1: Localización del entorno de estudio del rio Manzanares en Madrid

Fuente: Elaboración propia (2020)

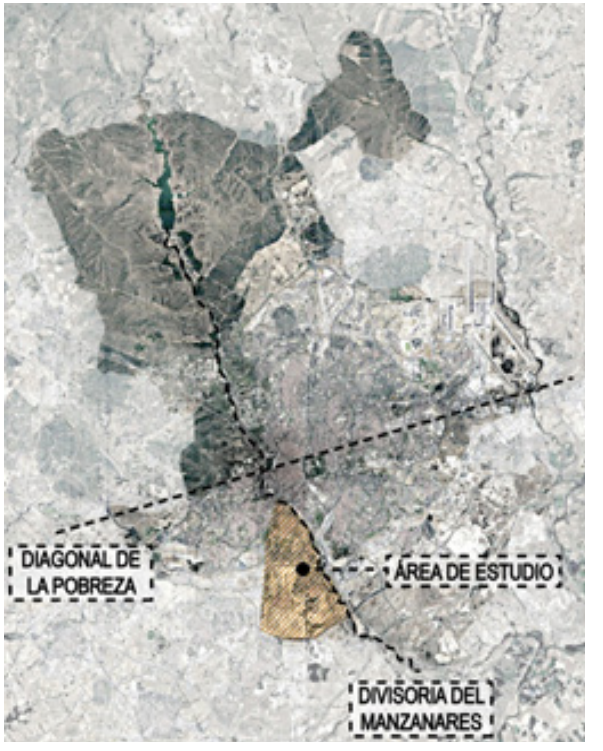

Figura 2: Diagonal de la pobreza y diagonal del Manzanares en Madrid

Fuente: Elaboración propia (2020) 


\section{Estado del arte}

La clave para comprender la estrategia medioambienta de la cuenca sur del Manzanares reside en el comienzo del Parque Lineal (diseñado por Ricardo Bofill en el año 2000), señalado en el elemento 3 de la Figura 3, ya que en la actualidad la conexión con la cuenca media se limita a una pasarela elevada que conecta caminos de tipo marginal: el nodo de la M-30 (Plata y Ibarra Vega, 2016). Una estrategia de restauración medioambiental puede transformar este espacio en un nodo de infraestructura verde para el tránsito y usabilidad peatonal, que conviva ambientalmente con el nodo existente de la M-30.

En el Parque Lineal también se encuentran dotaciones de movilidad que fomentan la interacción entre los habitantes de los distritos con la franja verde de Manzanares, así como de la conexión peatonal entre los distritos que recorre. De todas ellas, la única que no tiene una función de tránsito es la instalación deportiva de "La Caja Mágica", representada en la Figura 4, actualmente conocida como "Centro Deportivo Multifuncional de Parque del Manzanares" (Figuras 3 y 4 , elemento 5). Este proyecto fue ejecutado para potenciar la candidatura madrileña a los Juegos Olímpicos de 2012 y 2016 (Molina Costa y Rey Mazón, 2011). El proyecto original incluyó, además de pabellones deportivos, un subproyecto que contemplaba la recuperación del entorno del paseo de Manzanares que circulaba junto a las instalaciones. Sin embargo, esta dotación se encuentra infrautilizada y todavía no está cumpliendo las expectativas de mejora con respecto a la usabilidad, movilidad y relaciones sociales ni de los vecinos de Usera ni de Villaverde. Es más, en los últimos años ha sufrido un deterioro que ha supuesto una carga para el barrio:

(...) con el tiempo se ha demostrado infrautilizada [La Caja Mágica] y con un gran coste de recursos. Además, el diseño de parque se encuentra poco adaptado a un ecosistema de ribera paseos de madera, rectilíneo) que con el tiempo se ha visto un deterioro incipiente. (..) Por ello, es necesario revertir estos planteamientos y retomar soluciones más sostenibles y que se apoyen en el carácter ribereño del parque dinamizando sus potencialidades (Martínez Escobar, 2019, p. 68).

Entre los aspectos más representativos del deterioro de las infraestructuras ejecutadas, y de acuerdo con las demandas vecinales, se destacan con respecto a la salubridad:

- Suciedad, basura y falta de mantenimiento presente en los parques y paseos debido a la falta de civismo y sobrecarga de los técnicos municipales ante el abandono de escombros y electrodomésticos.

- Falta de control de plagas como moscas, mosquitos, cucarachas, hormigas y roedores en general.

- Olor desagradable proveniente del agua estancada o de agua en tratamiento de las EDAR (Estación Depuradora de Aguas Residuales).

Paralelamente, se encuentran los relativos a la usabilidad, sobresaliendo las siguientes afecciones experimentadas por la vecindad:
- Espacios infradotados de alumbrado público dificultando su usabilidad al anochecer y a la madrugada.

- Escasa presencia de papeleras, o que favorece la suciedad dispersa por falta de civismo de sus usuarios.

- Espacios con una conectividad discontinua e infradotada en los pasos rápidos de circulación de vehículos, lo que dificulta su uso lineal.

- Presencia de espacios mixtos, donde, en determinadas franjas horarias, deben convivir diferentes actividades incompatibles, como son: las activas - travesías en bicicleta o carrera deportiva - y las pasivas - pasear caminar, juegos infantiles, actividades con mascotas, descanso para personas mayores, dotaciones, etc. -

- Falta de accesibilidad universal y diseño para todos, con un gran número de tramos de escaleras y falta de referencias que faciliten la orientación.

A su vez, en el tramo inferior del EDAR de Butarque se disipa la inversión y recuperación ambiental, empeorando en las zonas de transición entre municipios. Este aspecto es significativo porque, nuevamente, los límites municipales condicionan la salubridad y seguridad de los ciudadanos. $Y$ este aspecto se vuelve especialmente notable en el entorno residencial de Perales del Río (perteneciente a municipio de Getafe), en el que el vertido de escombros y basura convive con el abandono en el río de coches robados, contaminando el tramo bajo del Parque Lineal y Regional del Sureste (Martínez Escobar, 2019).

Aunque los problemas que representan riesgos sobre la salubridad están controlados por los organismos municipales mediante partidas de mantenimiento $y$ tratamientos larvicidas y desratización, en los últimos años los vecinos han experimentado un aumento en los riesgos, especialmente por insectos.

En el caso de Madrid, es el gobierno de la comunidad quien gestiona individualmente con los ayuntamientos estas acciones (Orsolich, 2011). Sin embargo, los límites de los ecosistemas lineales, como son los ríos, no pueden ser gestionados desde una perspectiva municipal, requiriendo de una organización política superior que coordine las acciones de los Municipios de Madrid y Getafe. Con ello, se podrían evitar desfases entre las agendas de actuación y los ciclos climáticos que sufren ambos municipios, reduciendo la posibilidad de aparición de los riesgos sanitarios denunciados por los vecinos. Actualmente, las plagas (de insectos y roedores, destacando la mosca negra y los mosquitos) han sufrido un incremento debido, en parte, a las variaciones climáticas y a que los núcleos urbanos proporcionan un ecosistema proclive para su desarrollo. Por ello, y siguiendo la estrategia de desinfección vírica y bacteriológica, se han logrado reducir los avisos a lo largo de la cuenca del río Manzanares en el distrito de Villaverde (Tamayo, Cámara y Escobar, 2013).

Asimismo, existe riesgo bacteriológico y vírico ligados a encharcamiento o acumulación de aguas contaminadas (Pérez-Gutiérrez y Pérez-Pérez, 2006), las cuales precisan de un mantenimiento específico. Además de la renovación y mejora de las instalaciones de tratamiento 
de aguas, el Ayuntamiento de Madrid comenzó en 2016 a abrir las compuertas de las cuencas medias y bajas para generar corrientes que evitaran el estancamiento. Esta acción también permite la incorporación de nuevas especies y la regeneración del ecosistema fluvial, tan necesario para que se produzca un control sanitario natural y pasivo de las plagas.

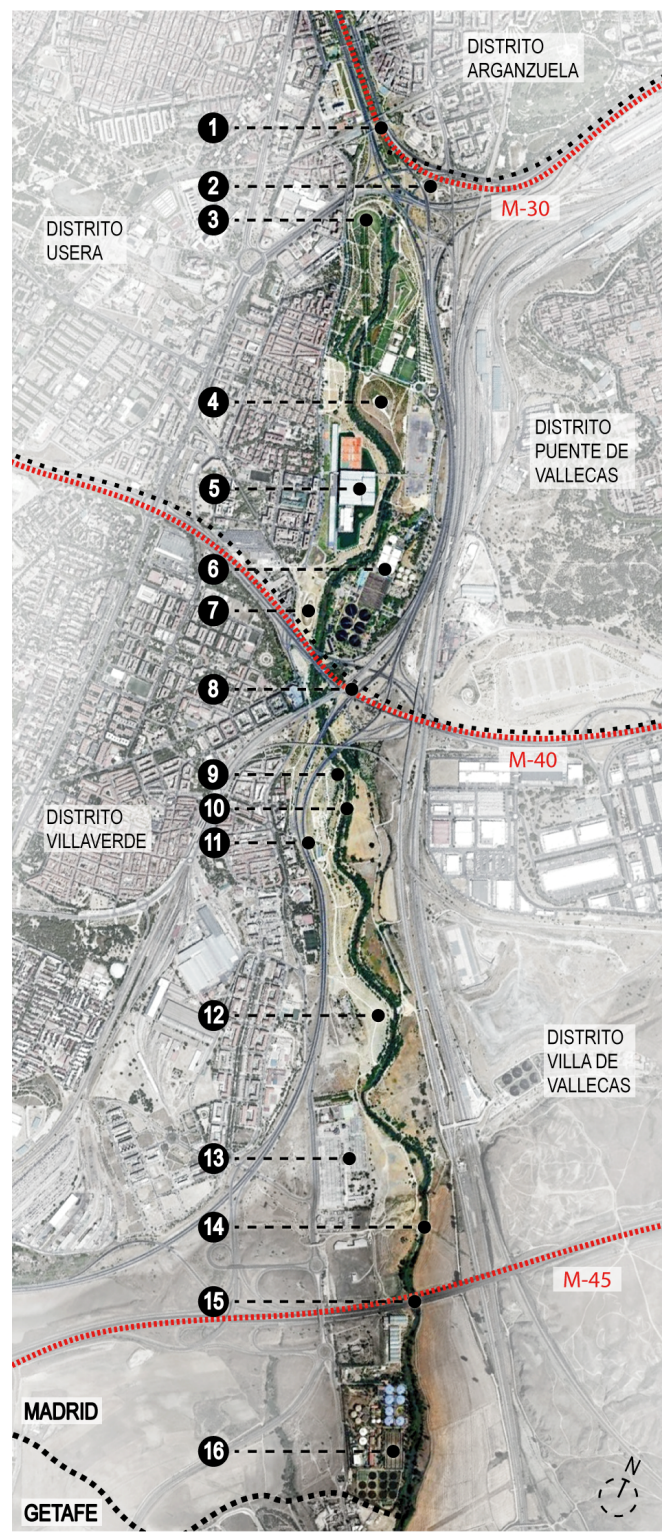

1. Pasarela peatonal nudo sur. 2. Nudo sur (m-30). 3. Cabecera del Parque Lineal de Ricardo Bofill. 4. Mirador del Parque del Rio Manzanares. 5. Complejo deportivo "La Caja Mágica". 6. EDAR La China. 7. Parque Dinosaurio. 8. Nodo M-40. 9. Parque Fluvial del Rio Manzanares. 10. Ribera del Rio Manzanares. 11. Entrada bajo vias a la altura de la C/Concepción de la Oliva. 12. XX. 13. Subestación eléctrica. 14. Pasarela del Trasvase Fluvial. 15. Barrera M-45. 16. EDAR Butarque.

Figura 3: Cuenca sur del río Manzanares correspondiente al municipio de Madrid

Fuente: Elaboración propia (2020)

Esta última actuación forma parte del Plan de Regeneración del Manzanares que se sigue ejecutando en la actualidad. Destacan el éxito que está suponiendo frente a los riesgos sanitarios detectados, debido principalmente a que, al aumentar la biodiversidad, la competencia natural de las especies regula su propio crecimiento y desarrollo.
Siguiendo el Plan de 2016, se están realizando diferentes campañas de control de roedores que se están extendiendo a los campos circundantes de los Distritos Sur. Estas campañas pretenden no solo controlar los roedores urbanos más comunes, como ratones y ratas, sino que también se han extendido para el control de conejos y liebres que están actuando como vectores de diferentes enfermedades de transmisión animal a humanos. Ante los riesgos sanitarios que supone la transmisión de enfermedades, las agrupaciones de veterinarios han puesto en conocimiento de las autoridades la exposición de los vecinos a una posible transmisión animal-humano a través de sus mascotas. El riesgo se acentúa en espacios como los que presentan las zonas de ribera Madrid Río y Parque Lineal, muy cercanos a focos potenciales de enfermedades.

Este proyecto también se engloba en la estrategia de restitución medioambiental sur en el que el municipio de Getafe se podrá conectar a Madrid mediante una infraestructura verde denominada Getafe Río, que recuperará las riberas del Manzanares a su paso por Perales del Río. Con esta intervención se prevé, además un aumento tanto del mobiliario urbano de bancos y papeleras, como de la limpieza y usabilidad del entorno.

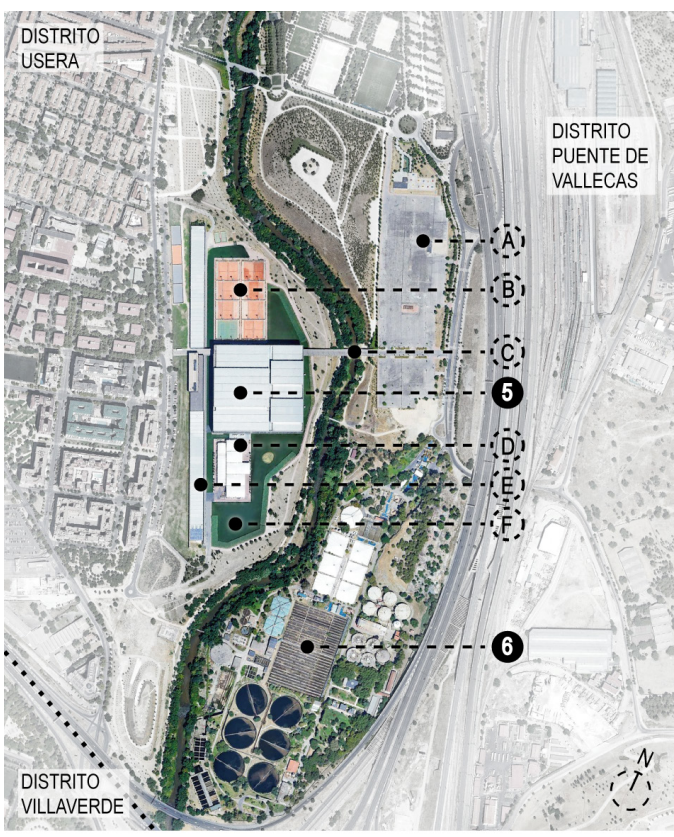

5. Complejo deportivo "La Caja Mágica". A. Tanque de Tormentas y usos múltiples. Tenis Garden. C. La pasarela. D. Edificio Tenis Indoor. E. Media Garden. F. Lago ecológico. 6. EDAR La China.

Figura 4: Detalle Complejo deportivo "La Caja Mágica" Fuente: Elaboración propia (2020)

No obstante, esta actuación derivó en otras dotaciones pasivas relacionadas con la salubridad y seguridad medioambiental de gran relevancia, como son:

- Un tanque de tormentas (Figura 4, elemento A) situado por delante de la entrada al EDAR de La China (Figura 4, elemento 6) y paralelo a "La Caja Mágica" (Figura 4 elemento 5). Su función es doble: la primera es regular y tratar de forma primaria el flujo de agua resultante del 
exceso de lluvia o de las lluvias torrenciales; la segunda es emplear su superficie exterior como una explanada de usos múltiples y recreativos, como son festivales de música, el teatro al aire libre, etc.

- Un lago ecológico (Figura 4, elemento F) en forma de balsa de tormenta alrededor del edificio que filtra $y$ regenera el agua proveniente de la reurbanización de La China. Parte de esta agua se utiliza para regar los jardines del complejo, y el sobrante se vierte de vuelta al río. La depuradora de La China se construyó entre los años 30 y 50 (con un parón por la Guerra Civil), ya que todos los vertidos de la ciudad de Madrid se volcaban directamente al río Manzanares (Muñoz Serrano, 2017).

- Zona de parque vecinal en la ribera del río, con continuación de la arboleda presente en el camino de río Manzanares.

Como dotaciones activas de la salubridad y seguridad medioambiental se destacan las infraestructuras de las dos Estaciones Depuradoras de Aguas Residuales (EDARs). La más relevante, o que más carga recoge debido a su posicionamiento en el cauce del río, es La China (Figura 3, elemento 6). Ésta se encuentra al fina de su vida útil, similar a lo que sucede con la estación de Butarque (Figura 3, elemento 16), que precisa de inversiones para su renovación y mantenimiento. Dentro del plan de mejora y ampliación de las infraestructuras de saneamiento del río Manzanares se encuentra la construcción de un nuevo colector de refuerzo, que aliviaría la carga que recogen actualmente las estaciones de La China y Butarque (Ministerio para la Transición Ecológica y el Reto Demográfico, 2020).

Estas acciones han supuesto una inversión municipal y de la comunidad considerable y han resultado en una acción estratégica global que acompaña al Plan de Renaturalización del río Manzanares (da Silva Faustino, 2018). Sin embargo, todos los esfuerzos de inversión, tanto infraestructurales como dotacionales, parecen no terminar de conectar ni con las necesidades medioambientales de la ribera, ni con las demandas vecinales que buscan una mayor usabilidad de los espacios verdes, en especial para aquellos residentes de distrito de Villaverde y del barrio de Entrevías.

No obstante, esta estrategia de renaturalización también engloba la restitución medioambiental sur con la que el municipio de Getafe se podrá conectar a Madrid mediante una infraestructura verde combinada entre el Parque Lineal y Getafe Río, que recuperará las riberas del Manzanares a su paso por Perales del Río. Con esta intervención se prevé, además, un aumento dotaciona tanto del mobiliario urbano de bancos y papeleras, como de la limpieza y usabilidad del entorno.

\section{Problemáticas principales}

Acorde a las reuniones vecinales realizadas por el grupo de investigación Madrid Borde Sur, se han considerado todos los aspectos que se han señalado como riesgo para la restitución ambiental del río, su usabilidad y su salubridad. Estos aspectos son:
- Desconexión del paseo del Manzanares, entre Madrid Río y Parque Lineal, en el cruce de la M-30.

- Túneles por debajo de las vías para acceder al parque del Manzanares a la altura de C/Concepción de la Oliva que generan cierta inseguridad.

- Proyectos de intervención aislados. (Parque Lineal y Caja Mágica en Usera- San Fermín).

- Necesidad inmediata de renovación de las estaciones de depuración e inclusión de nuevas balsas de tormenta.

- Malos olores derivados de la falta de mantenimiento y de la necesidad de actualizar las estaciones de depuración.

- Instalación de asentamientos ilegales y vertidos no controlados en el tramo cercano a Perales del Río.

- Desconexión del Parque Fluvial con el paseo de Perales del Río, dificultando el paso entre municipios.

No obstante, tras analizar el estado del arte, se pueden identificar ciertas diferencias entre las demandas vecinales y las actuaciones estratégicas ejecutadas. Se destacan cuatro problemáticas principales que deben ser atendidas desde el marco estratégico de la restauración de la cuenca sur del Manzanares:

- La falta de conectividad del nudo norte.

- La falta de accesibilidad en el conjunto de trazado.

- La falta de usabilidad por motivos ambientales y de salubridad.

- Falta de conectividad entre el interior de los distritos y el Parque Lineal.

Estas problemáticas representan una oportunidad de mejora, ya que se proyecta una mayor usabilidad y accesibilidad en las futuras medidas que se ejecuten. Por otra parte, las medidas ambientales ya comienzan a obtener resultados positivos con respecto a la salubridad y se espera que, progresivamente, la restitución vegetal incremente el valor ecológico del cauce fluvial. Con ello aumentará de manera pasiva el control sanitario.

El nudo norte se caracteriza por ser una conexión rápida de las vías de circulación principales de las carreteras: M-30, A-4 y de la Avenida de Andalucía, tal y como se aprecia en la Figura 5. Esta infraestructura representa una barrera tanto al libre tránsito peatonal como al de especies animales fuera de riesgo. No obstante, existen pasos que permiten el tránsito, aunque su usabilidad es baja por la concentración de contaminantes acústicos, atmosféricos, encharcamientos $y$, principalmente, porque no permite la accesibilidad universal del espacio para todos los ciudadanos.

A su vez, es importante destacar que esta zona dispone de un proyecto en ejecución con un presupuesto de 1.150.000 € para favorecer la accesibilidad mediante un trazado de un corredor de: $1,4 \mathrm{~km}$ de recorrido por 2,5 metros de ancho y con una pendiente máxima de $0,9 \%$, en el que se continuará con un uso mixto de 


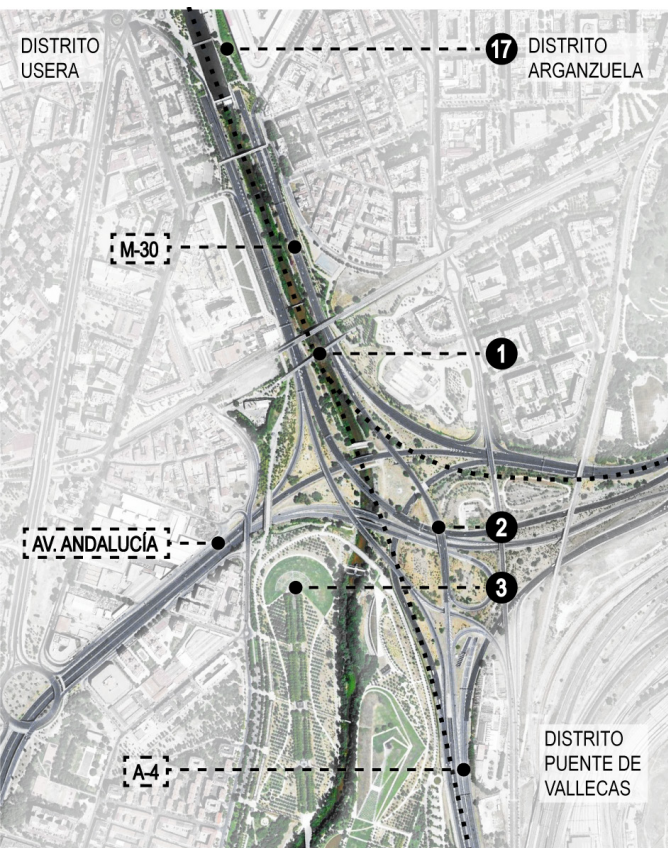

1. Pasarela peatonal nudo sur. 2. Nodo Sur M-30. 3. Cabecera del Parque Lineal de Ricardo Bofill. 17. Madrid Rio.

Figura 5: Nudo Sur M-30

Fuente: Elaboración propia (2020)

usuarios entre peatones y deportistas, dando comienzo en la presa 9 de Madrid Río, y conectando la actuación de referencia con el Parque Lineal.

El objetivo, por tanto, del presente artículo radica en la búsqueda de los elementos críticos que presenta el parque lineal en la actualidad, con el fin de detectar posibles puntos de mejora para el mismo. Con ello se pretenderá buscar una solución que integre los pilares de la presente investigación (accesibilidad, usabilidad y salubridad), de manera que la estrategia de restauración ambiental de la cuenca sur del río Manzanares culmine como un proyecto de gran relevancia para la zona, mejorando la calidad de vida de sus vecinos.

\section{Metodología}

Para afrontar el análisis de los riesgos contemplados por los vecinos y asociaciones, así como los riesgos de accesibilidad, salubridad y usabilidad que se recogen en el marco teórico y en plan estratégico de la restauración ambiental del río, se ha realizado un estudio de campo desde noviembre de 2019 hasta julio de 2020. En él se especifican los factores de riesgo reales entre la conexión del nodo norte y el trazado de circulación hasta la cuenca sur de la M-40, y se han considerado los factores ambientales y morfológicos que afectan a la usabilidad del parque por parte de los vecinos de los distritos afectados.

Durante la realización de estos estudios, se llevaron a cabo diferentes consultas a modo de entrevistas (126 en total) a los usuarios de ambos tramos del Parque Lineal para poder poner en contexto los principios de la accesibilidad, usabilidad y salubridad recogidos por el

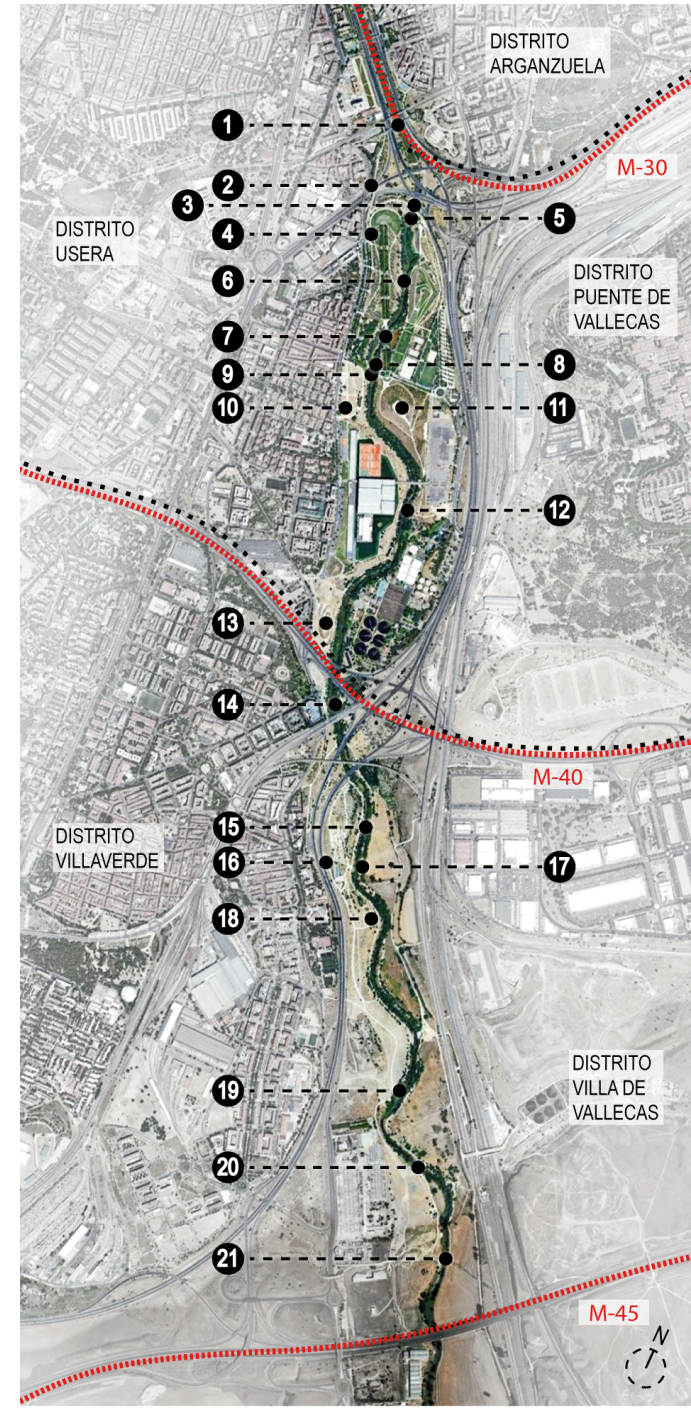

1. Pasarela de conexión del Paseo de la Ribera del Sur y Parque Lineal del Manzan res. 2. Pasarela peatonal de conexión entre el Parque Lineal Manzanares y Madrid res. 2. Pasarela peatonal de conexion entre el Parque Lineal Manzanares y Madrid Rio. 3. Pasarela tráfico rodado norte entre el Parque Lineal este y oeste. 4. Paseos del Parque Lineal del Manzanares. 5. Pasarela de Las Cascadas. 6. Pasarela peatonal central de conexión entre el Parque Lineal este y oeste. 7. Pasarela peatonal sur de conexión entre el Parque Lineal este y oeste. 8. Pasarela peatonal Puerta Manzanares. 9. Pasarela peatonal del Paseo Fluvial. 10. Zona verde junto al Parque Infantil de San Fermín. 11. Mirador del Parque Lineal del Manzanares. 12. Pasarela peatonal media (a la altura de la Caja Mágica). 13. Parque Dinosaurio del Manzanares. 14. Pasarela peatonal media (a la altura del Edificio Novosur). 15. Parque Lineal del Manzanares (zona sur). 16. Acceso al Parque Lineal bajo Autovía del Sur (A-4), a la altura de $\mathrm{C} /$ Concepción de la Oliva. 17. Pasarela peatonal media (a la altura del acceso al Parque Lineal bajo Autovía del Sur A-4). 18. Paseo Fluvial del Rio Manzanares. 19. Pasare peatonal sur (previo a la subestación éctrica) 20. Manzana Par 19. Pa Pasarela peatonal sur (a la altura de la subestación eléctrica). 21. Pasarela del
Trasvase Paseo Fluvial del Manzanares).

Figura 6: Puntos de referencia del análisis de campo Fuente: Elaboración propia (2020)

marco teórico y concretar las problemáticas expuestas por los vecinos del Distrito de Villaverde. Las 126 entrevistas se realizaron a lo largo de todo el recorrido del parque lineal, organizando los recorridos en grupos de 6 usuarios guiados por el técnico investigador, de diferentes edades y géneros, para obtener un relieve fehaciente en las percepciones problemáticas.

Las entrevistas representan una oportunidad única de poner en contexto cuantitativo las demandas vecinales 
frente a las actuaciones realizadas por la administración. Estas visitas de campo permitieron, por un lado, detectar los elementos clave en la extensión del Parque Lineal y, por otro lado, elaborar una valoración de los tres pilares del proceso de regeneración urbana: la accesibilidad, la usabilidad y la salubridad. De esta manera, los itinerarios se definieron siguiendo las actuaciones de renaturalización y urbanización ejecutados, permitiendo la extracción de conclusiones para cada uno de los tramos e hitos principales. Estos quedan recogidos en la Figura 6, repartidos a lo largo de todo el recorrido y señalando aquellos lugares donde los vecinos podían localizar mayores conflictos o, por el contrario, aquellos espacios donde se sentían más cómodos.

Tras definir los puntos de referencia, se elabora una valoración del investigador que contrastaba con 6 usuarios de diferentes edades y géneros. De esta forma se recogieron las percepciones de todos ellos en los conjuntos fundamentales con respecto al trazado planificado.

Las entrevistas recogieron la valoración de los vecinos en torno a las cuestiones de accesibilidad, salubridad y usabilidad, listadas en el marco teórico, para cada uno de los puntos localizados. Las valoraciones perceptivas y experienciales de cada uno de los voluntarios se tradujeron a una puntuación numérica con el fin de obtener una puntuación global para cada uno de los criterios señalados. Las valoraciones asociadas fueron las siguientes:

- 0 puntos: cuando el criterio a evaluar se encontraba en mal estado, con mal mantenimiento, o no cumplía los estándares mínimos para un uso adecuado del mismo.

- 1 punto: cuando el criterio evaluado se encontraba en un estado aceptable, pero con amplia posibilidad de mejora en cuando a calidad, estado o mantenimiento.

- 2 puntos: cuando el criterio presentaba un buen estado de conservación y limpieza, y además presentaba una buena calidad de uso.

Este modo de puntuación permitió la organización de los datos en tres estados fundamentales de malo, regular y bueno. Se pueden consultar los resultados analíticos enmarcados en la Tabla 1, donde se hace un resumen comparativo de los mismos, apoyándose además en un criterio de colores asociado a cada uno de los estados:

$$
\begin{aligned}
& \text { - Malo - rojo } \\
& \text { - Regular - naranja } \\
& \text { - Bueno - verde }
\end{aligned}
$$

Estos resultados se presentan en forma de matriz con referencia a 3 criterios estipulados para los 3 pilares del estudio: la accesibilidad, la usabilidad y la salubridad:

- Accesibilidad (vecinos de la tercera edad, personas con discapacidad física y personas con discapacidad visual)

- Usabilidad (manejabilidad, zonas verdes y conectividad)

- Salubridad (polución, iluminación y contaminación, incluyendo la acústica)
Estas valoraciones se completaron con los comentarios específicos aportados tanto por los técnicos investigadores como por los entrevistados.

Por último, estas entrevistas también condujeron a la realización de un análisis donde se pusieron en relación los espacios dotacionales interiores de los barrios con el propio del Parque Lineal. Además de todas las problemáticas que se plantearon durante el encuadre del marco teórico, se detecta una nueva amenaza en cuanto la necesidad de inversión para evitar la fragmentación socioeconómica de sus ciudadanos. Este reto estratégico persigue que puedan producirse mejoras en el barrio con el fin de que tanto el interior de los distritos como el resto de los madrileños puedan disfrutar de las mejoras medioambientales del río Manzanares.

Por ello, tras analizar con detenimiento todas las propuestas y actuaciones municipales, se identifica una falta de correlación estratégica entre el interior del distrito y del eje medioambiental del río Manzanares en el Parque Lineal. Este aspecto supone uno de los principales motivos por los que los vecinos y las asociaciones vecinales demandan atención a los distintos grupos municipales. Ya que, al focalizar todas las estrategias en el margen fluvial, se han reducido las inversiones que pudieran conectar, mediante vías o infraestructuras verde, los espacios degradados o abandonados del interior del distrito con el del río.

Para analizar este efecto, también se han puesto en relieve todos los espacios dotacionales del Parque Lineal con el interior de los distritos, con el fin de determinar posibles conexiones que favorezcan la interacción entre las áreas barriales interiores y las zonas verdes de ribera, ya que nuevamente esta falta de correlación entre el interior edificado y la urbanización de recuperación medioambiental supone una oportunidad de intervención para mejorar la usabilidad y calidad de vida de los vecinos de Villaverde. Por ello se ha realizado un análisis metodológico complementando las problemáticas identificadas por la ciudadanía del distrito, unificándolas con la estrategia de renaturalización. De esta forma se han podido identificar los puntos verdes clave del distrito para aumentar la conectividad y mejora de la movilidad, usabilidad y salubridad de los espacios intermedios (solares en desuso o sin edificación).

\section{Resultados y hallazgos principales}

Tras el análisis de los 21 puntos clave del itinerario del Parque Lineal, recogidos en la Tabla 1, se destaca que, en general, la accesibilidad, usabilidad y salubridad es buena, a excepción de:

- Punto 1. Pasarela de conexión del paseo de la ribera sur, el cual circula sobre parte del viario del Nodo M-30.

- Punto 11. Mirador del Parque Lineal del Manzanares, donde no existen buenas condiciones de usabilidad y accesibilidad debido a su posición sobre una colina. 
- Punto 13. Parque del Dinosaurio del Manzanares, el cual se coloca junto al EDAR La China y posee propiedades materiales y ambientales que dificulta su usabilidad tanto en invierno como en verano.

Estos puntos tienen una valoración regular y mala, y precisan de mantenimiento y obras de consolidación. Por otra parte, se identifican los puntos 3,10 y 16, que necesitan una mayor atención, ya que presentan una valoración mayoritariamente negativa en los tres aspectos fundamentales. Su mejora es por tanto una necesidad para poder consolidar la actuación integral a lo largo del Parque Lineal. Estos tres puntos son:

- Punto 3. Pasarela Rótula Norte entre Parque Lineal y Madrid Río, que circula sobre el viario que conecta la M-30 con otras vías de alta velocidad.

- Punto 10. Zona verde junto al Parque Infantil de San Fermín, la cual presenta una gran desconexión con respecto al Parque Lineal, creando problemas de acceso.

- Punto 16. Acceso al Parque Lineal bajo la Autovía del Sur A-4, donde existen condiciones ambientales desfavorables debido a su posición respecto a la vía de alta velocidad.

De todos estos aspectos se destaca la mejora medioambiental generalizada del río, ya que ha supuesto una reducción de los riesgos sanitarios por el estado de conservación del río, destacando que el tramo analizado no presentaba problemas de plagas durante el proceso de análisis y, por tanto, se puede determinar que las acciones que se están llevando a cabo son efectivas y coherentes con el objetivo del proyecto de renaturalización.

Además, se ha realizado el análisis de espacios vacantes con potencial, recogido en la Figura 7, para para crear una red de espacios verdes que pongan en valor las inversiones realizadas en el Parque Lineal, y las conecten con el interior del distrito.

Este análisis de los barrios interiores del Distrito de Villaverde supone un avance para poder visualizar el potencial de conexión perpendicular a la estrategia ambiental del Manzanares. Esta conexión perpendicular debe formar parte de la revisión estratégica, ya que mediante unos corredores verdes interiores, como se muestran en la Figura 8, se conectarían los barrios con mayor división socioeconómica y con mayor sensación de abandono municipal.

\section{Discusión}

Las consultas realizadas a los vecinos derivaron en la reivindicación de una problemática social existente con respecto a la movilidad y conectividad de los barrios interiores con respecto a las actuaciones que se llevan a cabo en el Parque Lineal y resto de Madrid. Este aspecto cobró gran relevancia durante una reunión con la Asociación de vecinos Nave Boetticher, donde clarificaron el sentimiento de abandono vecinal que experimentan por parte de las autoridades municipales en relación a los problemas sociales estructurales de los barrios interiores del distrito.
El análisis metodológico denota que las demandas sociales de los habitantes de Villaverde con respecto a la estrategia ambiental del Manzanares se encuentran en distintas fases de consecución. Así, recogemos los aspectos problemáticos de los ciudadanos del distrito, como son:

Estado medioambiental del río. Todavía faltan intervenciones que consigan crear una infraestructura verde continua entre la cuenca media y la cuenca sur.

- Riesgos sanitarios por el estado de conservación del río y sus márgenes. La falta de inversión municipal y la falta de coordinación entre los municipios del sur.

- Movilidad. Es necesaria una intervención que mejore la usabilidad y la transitabilidad de los parques existentes, cosiendo los tejidos interiores con las zonas dotacionales junto al río.

Fragmentación socioeconómica del Distrito de Villaverde. Una mejora de la infraestructura verde ayudará al desarrollo económico de la zona, reduciendo las diferencias que existen actualmente entre los distritos afectados por Madrid Río y los de Parque Lineal.

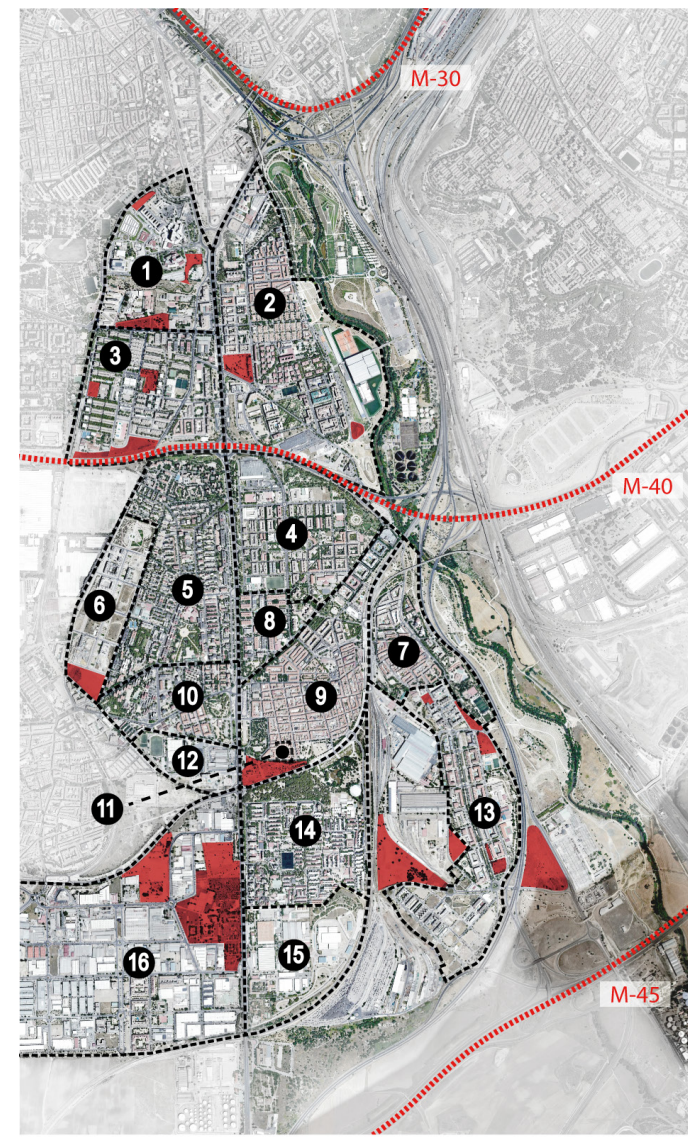

Solares intermedios

Barrios: 1. Hospital 12 de Octubre. 2. San Fermin. 3. Orcasur. 4. El Espinillo. 5. Ciudad de los Ángeles. 6. Barreiros. 7. Los Rosales. 8. Oroquieta. 9. Villaverde Bajo. 10. El Cruce .11. La Quinta del Pilar. 12. Botticher. 13. Butarque. 14. San Cristóbal. 15. Cuarteles. 16. Polígono de Marconi.

Figura 7: Solares intermedios en el interior de los distritos de Villaverde y Usera

Fuente: Elaboración propia (2020) 
Miguel Ángel Ajuriaguerra Escudero / Alba Ramírez Saiz

\begin{tabular}{|c|c|c|c|c|c|}
\hline \multicolumn{6}{|c|}{ ANÁLISIS DEL ESTADO DE LOS DISTINTOS PUNTOS Y ESPACIOS DE LA CUENCA SUR DEL RIO MANZANARES } \\
\hline \multicolumn{2}{|r|}{ Punto de referencia } & \multirow{2}{*}{\begin{tabular}{|l|} 
Acces. \\
A F V
\end{tabular}} & \multirow{2}{*}{\begin{tabular}{|c|} 
Usab. \\
M ZV C
\end{tabular}} & \multirow{2}{*}{$\begin{array}{l}\text { Salub. } \\
\text { P L CA }\end{array}$} & Comentarios \\
\hline 1 & $\begin{array}{l}\text { Pasarela de Conexión del Paseo de la } \\
\text { Ribera del Sur y Parque Lineal del Man- } \\
\text { zanares. Km 0. }\end{array}$ & & & & $\begin{array}{l}\text { El acceso a la pasarela se realiza a través de camino con bastante } \\
\text { pendiente. Esta construcción discurre sobre varios carriles de alta } \\
\text { velocidad, por lo que hay contaminación aérea, acústica y lumínica. }\end{array}$ \\
\hline 2 & $\begin{array}{l}\text { Pasarela peatonal de conexión entre } \\
\text { el Parque Lineal Manzanares y Madrid } \\
\text { Río. Km 0'5. }\end{array}$ & A F V & M Zv C & P L CA & $\begin{array}{l}\text { El acceso se realiza en llano en ambos extremos. Esta pasarela cir- } \\
\text { cula sobre carriles de alta velocidad. Existe contaminación aérea, } \\
\text { acústica y lumínica. }\end{array}$ \\
\hline 3 & $\begin{array}{l}\text { Pasarela tráfico rodado norte entre el } \\
\text { Parque Lineal este y oeste. Km } 0^{\prime} 75 \text {. }\end{array}$ & A F V & M zv C & P L CA & $\begin{array}{l}\text { El mantenimiento y conservación de esta pasarela es bajo, con } \\
\text { tablones de madera arqueados. Por ello, los peatones utilizan la } \\
\text { calzada de servicio para circular. }\end{array}$ \\
\hline 4 & $\begin{array}{l}\text { Paseos del Parque Lineal del Manzana- } \\
\text { res. Km 0'8. }\end{array}$ & A F V & M Z C & P L CA & $\begin{array}{l}\text { Buen mantenimiento y limpieza, con grandes espacios de sombra } \\
\text { en verano. Faltan referencias visuales contra la desorientación. Con } \\
\text { desniveles acusados sin barandilla. }\end{array}$ \\
\hline 5 & Pasarela de Las Cascadas. Km 1. & A F V & M zv C & PLCA & $\begin{array}{l}\text { Pasarela a nivel entre los dos extremos del cauce sobre una de las } \\
\text { presas del río Manzanares. }\end{array}$ \\
\hline 6 & $\begin{array}{l}\text { Pasarela peatonal central de conexión } \\
\text { entre el Parque Lineal este y oeste. Km } \\
1^{\prime \prime 3} \text {. }\end{array}$ & A F V & M zv C & P L CA & $\begin{array}{l}\text { Estrecha pasarela de madera tiene falta de mantenimiento y con- } \\
\text { servación, con tablones arqueados que dificultan el tránsito. Los } \\
\text { extremos se apoyan con pendientes variables notables. }\end{array}$ \\
\hline 7 & $\begin{array}{l}\text { Pasarela peatonal sur de conexión en- } \\
\text { tre el Parque Lineal este y oeste. Km } \\
1^{\prime} 5 .\end{array}$ & A F V & M Z Zv C & P L CA & $\begin{array}{l}\text { Espacio de tránsito ancho, permitiendo la circulación peatonal flui- } \\
\text { da. Falta de mantenimiento con tablones arqueados. Acceso con } \\
\text { notables inclinaciones. }\end{array}$ \\
\hline 8 & $\begin{array}{l}\text { Pasarela peatonal Puerta Manzanares. } \\
\mathrm{Km} 1^{\prime} 7 \text {. }\end{array}$ & A F V & M zv c & P L CA & $\begin{array}{l}\text { Presenta un espacio de tránsito ancho para peatones con tablo- } \\
\text { nes arqueados. Ambos extremos de la pasarela se apoyan sobre } \\
\text { pendientes bajas. }\end{array}$ \\
\hline 9 & $\begin{array}{l}\text { Pasarela peatonal norte del Paseo Flu- } \\
\text { vial } \\
\mathrm{Km} 1^{\prime} 75 .\end{array}$ & A F V & M Zv C & P L CA & $\begin{array}{l}\text { El paso se realiza a nivel, por lo que las pendientes son muy ligeras. } \\
\text { El estado de conservación de la barandilla es medio, presentando } \\
\text { desconchones de pintura. }\end{array}$ \\
\hline 10 & $\begin{array}{l}\text { Zona verde junto al Parque Infantil de } \\
\text { San Fermín. Km 1'8. }\end{array}$ & A F V & M ZV C & $P L C A$ & $\begin{array}{l}\text { Se encuentra fuera del Paseo Fluvial, con un acceso único por esca- } \\
\text { leras. La señalización del parque es deficiente. La masa arbórea es } \\
\text { insuficiente para su uso en verano. }\end{array}$ \\
\hline 11 & $\begin{array}{l}\text { Mirador del Parque Lineal del Manza- } \\
\text { nares. Km } 2 \text {. }\end{array}$ & A F V & M Zv C & P L CA & $\begin{array}{l}\text { El acceso hasta el mirador presenta grandes pendientes y, al llegar a } \\
\text { la zona superior, existen pocos espacios de descanso. Además, hay } \\
\text { escasa vegetación, no existiendo a penas sombra. }\end{array}$ \\
\hline 12 & $\begin{array}{l}\text { Pasarela peatonal media (a la altura de } \\
\text { La Caja Mágica). Km 2’5. }\end{array}$ & A F V & M Zv C & P L CA & $\begin{array}{l}\text { Esta pasarela se encuentra a nivel a ambos extremos del río, y pre- } \\
\text { senta un buen estado de conservación. }\end{array}$ \\
\hline 13 & $\begin{array}{l}\text { Parque Dinosaurio del Manzanares. } \\
\mathrm{Km} 3^{\prime} 1 \text {. }\end{array}$ & A F V & M ZV C & P L CA & $\begin{array}{l}\text { Tiene la depuradora La China al este y al Tanatorio M40 al oeste. } \\
\text { Se accede mediante escalera o rampa, a lo largo de un camino de } \\
\text { tierra. Al sur, se encuentra la Autopista de Circunvalación M40, por } \\
\text { lo que hay contaminación, aérea, lumínica y acústica. }\end{array}$ \\
\hline 14 & $\begin{array}{l}\text { Pasarela peatonal media (a la altura del } \\
\text { Edificio Novosur). Km } 3^{\prime} 5 \text {. }\end{array}$ & A F V & M ZV C & $P L C A$ & $\begin{array}{l}\text { La pasarela se encuentra a nivel en ambos lados del río, por lo que } \\
\text { presenta pendientes muy livianas. Se encuentra en buen estado de } \\
\text { conservación. }\end{array}$ \\
\hline 15 & $\begin{array}{l}\text { Parque Lineal del Manzanares (zona } \\
\text { sur). } \mathrm{Km} 4 \text {. }\end{array}$ & A F V & M ZV C & P L CA & $\begin{array}{l}\text { Grandes explanadas boscosas, con mobiliario abundante, e instala- } \\
\text { ciones deportivas e infantiles. Existen senderos de tierra compacta- } \\
\text { da con pendientes medias-altas. }\end{array}$ \\
\hline 16 & $\begin{array}{l}\text { Acceso al Parque Lineal bajo Autovía } \\
\text { del Sur (A-4), a la altura de C/ Concep- } \\
\text { ción de la Oliva. Km } 4^{\prime} 3 \text {. }\end{array}$ & A F V & M Zv C & $P L C A$ & $\begin{array}{l}\text { La aproximación a este espacio es complicada. Existe una buena } \\
\text { limpieza de este espacio, pero no una buena conservación, ya que } \\
\text { no hay iluminación. Al pasar por debajo de la Autovía del Sur (A-4) } \\
\text { hay bastante ruido. }\end{array}$ \\
\hline 17 & $\begin{array}{l}\text { Pasarela peatonal media (a la altura del } \\
\text { acceso al Parque Lineal bajo Autovía } \\
\text { del Sur A-4). Km 4'3. }\end{array}$ & A F V & M zv c & PL L CA & $\begin{array}{l}\text { La pasarela ofrece una conexión a nivel entre ambos lados del río. } \\
\text { Su estado de conservación es bueno. }\end{array}$ \\
\hline 18 & $\begin{array}{l}\text { Paseo Fluvial del río Manzanares. } \mathrm{Km} \\
4^{\prime} 5 \text {. }\end{array}$ & $A F V$ & M zv C & P L CA & $\begin{array}{l}\text { El lado derecho del río tiene grandes pendientes y el izquierdo pre- } \\
\text { senta desniveles livianos. Las grandes masas arbóreas facilitan su } \\
\text { uso en verano. }\end{array}$ \\
\hline 19 & $\begin{array}{l}\text { Pasarela peatonal sur (previo a la Sub- } \\
\text { estación eléctrica). Km 5'5. }\end{array}$ & A F V & M ZV C & P L CA & $\begin{array}{l}\text { Paso a nivel entre los dos extremos el rio. Presenta un buen estado } \\
\text { de conservación y un mantenimiento adecuado. }\end{array}$ \\
\hline 20 & $\begin{array}{l}\text { Pasarela peatonal sur (a la altura de la } \\
\text { Subestación eléctrica). Km } 6 \text {. }\end{array}$ & A F V & M Zv C & P L CA & $\begin{array}{l}\text { Paso a nivel entre los dos extremos el rio. Presenta un buen estado } \\
\text { de conservación y un mantenimiento adecuado. }\end{array}$ \\
\hline 21 & $\begin{array}{l}\text { Pasarela del Trasvase Paseo Fluvial del } \\
\text { Manzanares. Km 6'4. }\end{array}$ & A F V & M ZV C & $P \perp C A$ & $\begin{array}{l}\text { El camino de acceso a este puente del margen derecho tiene una } \\
\text { pendiente notable. El estado de conservación es bueno. }\end{array}$ \\
\hline & & & & & \\
\hline
\end{tabular}

Tabla 1: Análisis de puntos de referencia de la cuenca sur del río Manzanares en función de criterios de Accesibilidad, Usabilidad y Salubridad

Fuente: Elaboración propia (2020) 
Para conseguir una mejora de todas estas problemáticas se propone la creación de una red de espacios verdes a través de la reconversión de los solares intermedios vacíos existentes en la actualidad de la Figura 7. Esta estrategia busca resolver las cuatro problemáticas planteadas mediante la creación de corredores verdes internos, tal y como se señala en la Figura 8.

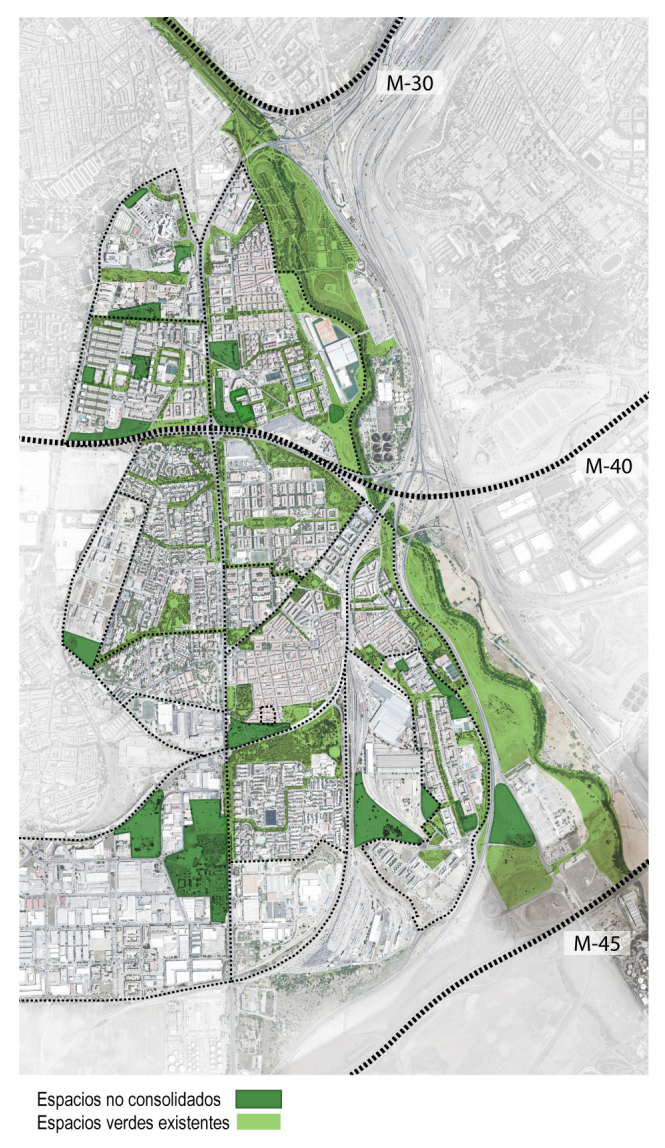

Figura 8: Propuesta de vías verdes para mejorar la conectividad del interior del distrito con el Parque Linea del río Manzanares

Fuente: Elaboración propia (2020)

Es importante valorar la estrategia de renaturalización de río Manzanares por el impacto ambiental tan positivo que ha supuesto con respecto a las mejoras y recuperación de ecosistemas del río. También hay que destacar el impacto que esta ha ejercido sobre los distritos del sur de la capital, tradicionalmente industriales, y especialmente con relación a las mejoras experimentadas en materia de accesibilidad, usabilidad y salubridad. No obstante, este impacto ha servido para incentivar procesos de gentrificación, primero en el tramo de Madrid Río, y actualmente en la parte alta del Parque Lineal. Estos aspectos dejan de lado a los ciudadanos de los barrios de los distritos del sur, que experimentan unas mejoras sustanciales en las proximidades de la cuenca del río, pero que en su interior siguen necesitando actuaciones que mejoren su accesibilidad, usabilidad y salubridad.

Esta investigación, realizada en el tramo bajo o sur de Manzanares, ha puesto en relieve los resultados de las inversiones municipales a lo largo de los últimos años. A pesar de ello, la ciudadanía y las asociaciones vecinales del Distrito de Villaverde continúan experimentando un abandono institucional que responde, principalmente, a la inversión focalizada en el perímetro del distrito, dejando sin recursos aparentes al resto de los barrios interiores. Este aspecto es destacable por el número de solares y parcelas no edificadas a lo largo del interior de distrito, que generan problemas de seguridad, usabilidad y salubridad. Son precisamente estos llamados espacios intermedios, u olvidados, como indica la ciudadanía, los que precisan de una reestructuración y ordenación que permitan la mejora de los aspectos indicados, y que, a la vez, mejoren el sesgo socioeconómico de sus vecinos mediante una mejor movilidad y relación con el resto de Madrid. Esta apreciación debería sumarse al Plan Estratégico del Manzanares mediante intervenciones que fomenten tanto una mayor movilidad entre el interior y el margen fluvial, como nuevas oportunidades empresariales dentro del distrito para aumentar la empleabilidad y la mejora salarial de sus ciudadanos.

Una de las propuestas actuales por parte del municipio es dotar uno de los solares sin uso del Barrio de San Fermín de un aparcamiento disuasorio. Esta medida resulta positiva con respecto a la consecución de los objetivos del proyecto Madrid 360 para la reducción de contaminación atmosférica. Sin embargo, no deja de ser una respuesta para los ciudadanos de los municipios del sur para acceder en vehículo privado a la red de transporte público del municipal, lo que ignora las necesidades de los propios vecinos de San Fermín. Por ello estas propuestas, como tantas otras que se están planteando, deberían tener en cuenta actuaciones cuyos principales beneficiados sean los propios vecinos y las necesidades de reestructuración social del conjunto. Con ello conseguirían un verdadero impacto en todos los aspectos que los vecinos del Distrito de Villaverde identifican como problemáticos

\section{Conclusiones}

Los distritos de Villaverde y Usera han presentado mejoras en la calidad de sus espacios dotacionales verdes gracias a la consolidación del eje del Parque Lineal. Es evidente que la regeneración medioambiental de este ha arrastrado consigo una mejora en la calidad de los equipamientos y los servicios públicos, lo que ha hecho que la calidad urbana de los barrios limítrofes aumente.

Sin embargo, la realidad es que este Parque Lineal presenta dos riesgos clave. Por un lado, la solidificación del parque se remonta a principios de los años 2000, lo que hace que sea necesario la rehabilitación y mantenimiento en algunos de los puntos más conflictivos, tal y como se ha detectado en las visitas de campo. Por otro lado, además, mientras que el parque ha supuesto una mejora de la calidad de vida para los residentes más cercanos, el interior de los distritos presenta una desconexión evidente en la movilidad con el parque.

Estos aspectos hacen que se vuelva imperativa una reestructuración de las dotaciones de espacio público en el interior de los barrios con el fin de conectarlos y atraerlos hacia el río. Pero, además, se debe procurar el mantenimiento y la continua mejora de las 
infraestructuras actualmente presentes en el parque con el fin de que la calidad del espacio no merme.

Asimismo, la cercanía a un gran número de vías hace que en muchos puntos del recorrido se produzca un exceso de polución y de contaminación acústica, resultando en una merma de la calidad de esta infraestructura verde. Este hecho, añadido a la presencia de espacios con alta pendiente $y / o$ barreras urbanísticas, hace que se reste confortabilidad al entorno del Parque Lineal.

Todos estos elementos de accesibilidad, usabilidad y salubridad son esenciales para garantizar que la regeneración que se llevó a cabo del río Manzanares sea eficiente y beneficiosa, tanto para el ecosistema fluvia como para todos los ciudadanos que viven junto a él.

\section{Agradecimientos}

Este trabajo no hubiese sido posible sin la colaboración de los vecinos anónimos que mostraron sus valoraciones durante las encuestas. También es muy importante agradecer los trabajos y disposición de todas las asociaciones vecinales contactadas en los últimos años, haciendo una especial mención a la Asociación de vecinos Nave Botticher, y toda su vinculación por proporcionar soluciones reales a todos los problemas vecinales. Asimismo, un especial agradecimiento a las diferentes administraciones que han atendido a este grupo de investigación para la realización de sus labores investigadoras.

Cómo citar este artículo/How to cite this article: Ajuriaguerra Escudero, M. y Ramírez Saiz, A. (2021). Estrategia de restauración ambiental en la cuenca sur del río Manzanares de Madrid Estoa. Revista de la Facultad de Arquitectura y Urbanismo de la Universidad de Cuenca, 10(20), 31-41 doi: 10.18537/est.v010.n020.a03

\section{Referencias bibliográficas}

Ajuriaguerra, M. A., Braojos Bueno, L. I., y López Parejo, A. (2019). Aristráin Villaverde, el menguante legado industrial del desarrollismo frente a la especulación en el sur de Madrid. Iniciativa Digital Politécnica.

da Silva Faustino, A. (2018). El río Manzanares en su paso por los tejidos urbanos de Madrid: El juego de posibilidades entre una biofilia hídrica cotidiana y la recuperación ecológica riparia. Territorios en formación (14), 20-39.

Martín Robles, I. y Pancorbo Crespo, L. (2014). El Madrid de Julio Cano Lasso. De la utopía a la realidad. Universidad Camilo José Cela.

Martínez Escobar, J. (2019). Dinámicas Territoriales en torno al Manzanares: Degradación y reivindicaciones de un ámbito marginal. Universidad Complutense de Madrid.

Ministerio para la Transición Ecológica y el Reto Demográfico. (2020). El MITECO y el Ayuntamiento de Madrid acuerdan encargar a la sociedad estatal ACUAES la ejecución de las depuradoras de La China, Sur y Butarque. Gobierno de España.

Molina Costa, P. y Rey Mazón, P. (2011). El urbanismo mágico: Megaproyectos en la era del despilfarro. Universidad de Valladolid.

Muñoz Serrano, B. (2017). Propuesta para la reconexión del nudo sur de Madrid, reconversión de la antigua estación depuradora" La China" en espacios de disfrute del agua [Tesis de Grado]. Universidad Camilo José Cela.

Orsolich, E. U. (2011). El Medio Ambiente Urbano en el Plan General de Madrid. Urban, (3), 80-110.

Orueta, F. D. (2015). Megaproyectos urbanos y modelo de ciudad. El ejemplo de Madrid Río. Cuaderno urbano. Espacio, cultura, sociedad, 19(19), 179-200.

Pérez-Gutiérrez, J. F. y Pérez-Pérez, F. (2006). Agua y medio ambiente. Observatorio Medioambiental, (9), 9-25. https://revistas.ucm.es/index.php/OBMD/article/ view/OBMD0606110009A

Plata, Á. M. e Ibarra Vega, D. (2016). Percepción local de estado ambiental en la cuenca baja del río Manzanares. Luna Azul, (42), 235-255.

Tamayo, I., Cámara, J. M. y Escobar, F. (2013). Gestión municipal de plagas urbanas. El caso de Madrid. Revista de Salud Ambiental, 13(1), 80-88. 